\title{
Introduction to the Special Issue on Gene-Hormone Interplay
}

\author{
K. Paige Harden ${ }^{1}$ Kelly L. Klump ${ }^{2}$
}

Received: 19 March 2015/ Accepted: 26 March 2015/Published online: 23 April 2015

(C) Springer Science+Business Media New York 2015

Hormones are messengers-signaling molecules that transmit information from cell to cell and from tissue to tissue (Cooper 2000). Unlike neurotransmitters, which travel only across the infinitesimal synaptic cleft, hormones can travel to distant tissues in the body, e.g., from the brain to the genitals and back again. The word "hormone" is derived from the Greek word for "impetus." Fitting that description, hormones are driving forces for growth and behavior; they impel and inhibit eating, drinking, mood changes, mating, fleeing, and fighting.

We chose the title "Gene-Hormone Interplay" for this Special Issue, because it is a general term that encompasses the variety of roles that hormones might play in the complex link between genotype and behavioral phenotypes. The most straightforward relationship between genes and hormones is simply that genes code for hormones, for hormone receptors, for hormone precursors, and for molecules necessary for hormonal synthesis, transport, and elimination. In fact, many of the specific genes involved in hormonal signaling have already been identified. For example, the $C R H$ gene codes for corticotrophin-releasing hormone, which is synthesized in and released from the hypothalamus as part of the hypothalamus-pituitaryadrenal-gland (HPA) cascade that ultimately results in the release of cortisol as part of the human stress response.

The behavioral genetic study of hormones is, in some ways, in the opposite position as the behavior genetic study of complex behavior. For cognitive ability or depression, we know generally how much population variation is due

Kelly L. Klump

klump@msu.edu

1 University of Texas at Austin, Austin, USA

2 Michigan State University, East Lansing, USA to genetic differences between people, but we are largely ignorant about which specific genes make up that effect. For hormones, we know which specific genes code for the key components of hormonal systems, but we are largely ignorant of whether variation in those genes accounts for much of the observed phenotypic variation in hormone levels. Two papers in this Special Issue (Van Hulle et al. 2015; Estourgie-van Burk et al. 2015) present data on the heritabilities of hormone levels during adolescence and are thus filling critical gaps in the literature.

One obvious but understudied question is if and how hormone-relevant genes are related to behavioral phenotypes. Illustrating this approach, two companion papers in this Special Issue examine the effects of a specific gene, Srd5al, which is involved in the synthesis of allopregnanolone (a neuroactive steroid that is synthesized from progesterone), on alcohol use behavior in an animal model (Ford et al. 2015; Tanchuck-Nipper et al. 2015). Two other papers use family designs to examine the links between hormonal processes and (latent) genetic risks for psychopathology. Marceau et al. (2015) use an adoption design to examine if genetic risk for psychopathology, as indexed by biological mothers' substance use, externalizing, and internalizing problems, is linked with children's morning cortisol levels. Additionally, Corley et al. (2015) use a longitudinal twin design to examine if genetic risk for adolescent substance use is linked with genetic influences on pubertal development.

Not only do genes code for hormones, but hormones regulate genes. In particular, steroid hormones (such as cortisol, estradiol, progesterone, and testosterone) bind to intracellular receptors that act as genetic transcription factors that directly regulate gene expression. For this reason, one emerging area of research is gene-by-hormone interactions: Do hormonal changes (such as experienced in 
puberty) activate or suppress the genetic influences on a behavioral phenotype via changes in gene expression? In this Special Issue, this question is explored in a paper by Corley et al. who test whether genetic influences on internalizing and externalizing symptoms increase after midpuberty.

In sum, hormones are potential mediators and moderators of genetic influences on behavior, yet behavioral genetic research on gene-hormone interplay is a field of study that is still in its infancy. This Special Issue presents a series of papers that present intriguing - and sometimes surprising-findings that offer an early glimpse into a burgeoning and important field of study. Spanning the lifespan from the prenatal period through middle adulthood, these papers point the way toward new avenues of discovery in behavioral genetic research.

\section{Prenatal period, infancy, and early childhood}

As described by Marceau et al., the prenatal environment has been hypothesized to shape the development of the fetal stress response system, with adverse in utero environments predicted to alter the responsiveness of the HPA axis. Similarly, early childhood stressors, such as harsh parenting, have also been hypothesized to "get under the skin" via their effects on HPA axis dysregulation. Marceau et al. use an adoption design to test whether (1) biological mothers' substance use and internalizing problems while pregnant, and (2) adoptive parents' overreactive parenting, predict children's morning cortisol, after controlling for lifetime indices of biological mothers' substance use, internalizing, and externalizing psychopathology. Their results underscore the importance of avoiding overly simplistic characterizations of the relationship between stress, cortisol, and psychopathology. Both adverse prenatal environments (i.e., in utero exposure to substance use and maternal depression) and adverse postnatal environments (i.e., adoptive fathers' overreactive parenting) were associated with higher morning cortisol levels at age 4.5 years. However, higher morning cortisol levels at age 4.5 years actually predicted lower internalizing problems at age 6 years, and morning cortisol was unrelated to externalizing problems. Marceau et al. interpret their results in terms of evolutionary theories that conceptualize cortisol as a biomarker of sensitivity to the environment, which can lead to either adaptive or maladaptive outcomes depending on context.

Estourgie-van Burk et al. also examine whether prenatal and early childhood environments have a lasting impact on hormonal systems. Previous research has found that children with restricted intrauterine growth (i.e., low birth weight) are at high risk for diabetes and cardiovascular disease in adulthood. In an effort to identify potential hormonal mechanisms for this persistent disease risk, Estourgie-van Burk et al. examine the relation between low birth weight and dehydroepiandrosterone sulfate (DHEAS, which is synthesized in the adrenal glands) and insulin-like growth factor I (IGF-I, which is synthesized in the liver following stimulation by growth hormone) in a sample of 18-year old twins and their siblings. They found that birth weight was associated with higher DHEAS and IGF-I levels, but these effects were apparent only in individuals who showed rapid "catch-up" growth in early childhood. By age 18, the preponderance of variation in both DHEAS and IGF-I levels was due to genes.

The papers by Marceau et al. and Estourgie-van Burk et al. reflect the growing scientific interest in examining hormonal systems as mechanisms for the biological embedding of early life experiences. They also note the challenges involved in disentangling the effects of the prenatal environment from genetic effects. Novel designs, such as those that focus on children conceived through assisted reproductive technology (e.g., egg donation, gestational surrogacy), may be helpful for future research in this area (Rice et al. 2009).

\section{Puberty and adolescence}

Adolescents are stereotyped as victims of "raging hormones," so it is no surprise that most of the papers in this Special Issue focus on puberty and adolescence. The study by Corley et al. highlights the complexity of understanding the genetic underpinnings of pubertal development, which involves changes in both adrenal hormones (e.g., dihydroepiandrosterone [DHEA]) and gonadal hormones (e.g., estradiol, testosterone). Their paper provides a comprehensive introduction to the study of individual differences in puberty, including level of development at any one point in time (pubertal status), the age at which one experiences a given level of development (pubertal timing), the velocity of pubertal changes (pubertal tempo), and the synchrony between changes involving different hormonal systems. The major strength of this study is its longitudinal design, incorporating annual measurements of pubertal development in twins from age 9 to age 15 .

Consistent with previous behavioral genetic research, they find substantial heritability-and limited effects of the shared environment-for pubertal timing in both boys and girls, regardless of how it was measured. However, their results caution against the assumption that various measures of pubertal development are necessarily always capturing the same underlying genetic signal, as unique genetic influences emerged for adrenarcheal timing versus gonadarcheal timing, for timing versus tempo, and for boys 
versus girls. Even seemingly minor modeling decisionssuch as how longitudinal data is combined to calculate pubertal tempo-can have large implications on estimates of genetic influence. Corley et al. also examine the genetic and environmental links between puberty and (1) substance use and (2) age at first sexual intercourse, and find the phenotypic associations to be largely attributable to genes. These results suggest that researchers interested in identifying specific genetic variants that confer risk for substance use should consider examining genes known to be involved in the hormonal events of puberty. Finally, this paper examines whether the heritability of internalizing and externalizing problems differs between adolescents who are preversus mid-puberty, as might be expected given potential hormonal effects on gene expression, but find largely null results.

The Corley et al. study posits that the genetic influences underlying individual differences in pubertal timing and tempo are related to hormonal processes, but the study does not directly measure hormonal levels. The study by Van Hulle et al., which measures DHEA, testosterone, and pubertal development in a cross-sectional sample of twins ages 12-16 years, is therefore an important contribution to the literature. They provide the first data on the heritability of DHEA in adolescents, as well as the first data on whether shared genes underlie the covariation between hormones from different endocrine systems (the adrenal hormone DHEA and the gonadal hormone testosterone).

They find evidence for heritable variation in testosterone for females only; for DHEA, the pattern was reversed, with greater heritable variation in males than in females, for whom there were moderate shared environmental influences. One particularly intriguing result from Van Hulle et al.'s multivariate analyses is that the covariation between DHEA and testosterone levels in male adolescents was primarily due to shared environmental variance; there were "few environmental influences specific to each hormone." This is consistent with previous work suggesting that both hormones are responsive to environmental stress and challenge, including adverse family environments. In discussing their results, Van Hulle et al. join Marceau et al. in noting the utility of genetically-informative designs for probing not just genetic but also environmental influences on hormonal functioning.

As they note in their paper, Van Hulle and colleagues' results for testosterone diverge from the few previous studies of testosterone in adolescence, which have found genetic influences on testosterone in both sexes or in males only. Additionally, their results for DHEA, particularly the finding of substantial shared environmental effects in males, appear to diverge from results presented in another paper in this Special Issue. Using a sample of 18-year-old twins, Estourgie-van Burk et al. present data on the heritability of DHEAS and IGF-I. Given that DHEAS and DHEA are each readily converted to the other, one might expect similar biometric results for the two hormones, but Estourgie-van Burk et al. found no evidence of shared environmental effects for DHEAS levels in either sex. Notably, all twin studies of hormones have used sample sizes that are small by the conventional standard of behavioral genetics; Van Hulle et al.'s sample size is the largest to-date with just over 300 twin pairs. Are individual differences in testosterone in adolescence heritable? Do the same genes influence other puberty-relevant hormones? Are the same genes involved in both males and females? Do genetic and environmental effects change with age? These are relatively straightforward questions, and the fact that there is not yet any clear consensus regarding their answers speaks to the need to invest in behavioral genetic research on hormones in larger sample sizes.

Using a younger, longitudinal sample of 9- to 12-yearolds, (Brouwer et al. 2015) examine whether hormonal changes in early adolescence are linked to structural brain changes and whether observed associations are due to genetic versus environmental influences. This study represents one of the first longitudinal examinations of genehormone-brain links. Underscoring the complexity of puberty-related hormonal change, their study focuses on a different suite of hormones than Estourgie et al. or Van Hulle et al., with measurements of luteinizing hormone (LH), follicle-stimulating hormone (FSH), estradiol, and testosterone. They find that, among girls, changes in FSH between ages 9 and 12 were correlated with changes in grey matter density throughout the brain. Interestingly, these hormone-brain associations were evident within $\mathrm{MZ}$ twin pairs: Non-shared environmental influences on FSH levels also influenced grey matter density in about half of the identified brain regions. Results for boys, however, were largely null. Overall, this study highlights how little we currently know about how the hormonal and neurobiological changes typical of early adolescence are related to each other or to genetic variation.

\section{Adulthood}

Adulthood is a developmental period that is often neglected when it comes to hormone research, as most studies focus on times of rapid hormonal changes (e.g., prenatal development, puberty). However, this focus misses a unique opportunity to understand hormonal variation during a relatively stable period of development during which one might expect to find the strongest gene-hormone or hormone-behavior associations.

Fortunately, three papers in this Special Issue, all using animal models, focus on adults and provide a glimpse into 
the window of opportunity that adulthood provides. In a pair of companion papers, Ford et al. and Tanchuck-Nipper et al. examine the effect of Srd5al deletion on alcohol consumption behaviors in mice. Srd5al codes for an enzyme necessary for the synthesis of allopregnanolone, a neuroactive steroid that is synthesized from progesterone and that positively amplifies agonist effects on $\mathrm{GABA}_{\mathrm{A}}$ receptors. Ford et al. find that deletion of the Srd5al gene results in changes in ethanol consumption, with the direction of the effect dependent upon both sex and ethanol access (continuous versus limited). In a companion paper, Tanchuck-Nipper et al. examine the acute effects of ethanol in mice; they also find sex-specific effects, with Srd5a1 deletion causing reductions in the anxiolytic effects of alcohol. Interestingly, Ford et al. note that the effects of a single gene may be mediated via multiple hormonal pathways, via "the accumulation of steroid precursors ... or the shunting of precursors to alternate biosynthetic pathways." In particular, they hypothesize that the deletion of Srd5al may alter cortisol and estradiol, which may in turn affect drinking behavior in sex-specific ways.

Ford et al. and Tanchuck-Nipper et al. begin with a single gene with a known hormonal function, and explore the effects of gene deletion on an array of behavioral phenotypes. In contrast, Blizard et al. begin with rat strains that have been selectively bred for behavioral differences, and explore whether these strains differ in hormonal systems. In particular, they focus on Maudsley Reactive rats, which have been bred for defecation in an open field, a behavioral model of stress responsiveness. Although research on biological stress response in humans has emphasized the role of the HPA axis, Blizard et al. found minimal differences between Reactive and Non-Reactive animals in either adrenocorticotropic hormone (ACTH) or corticosterone in response to an open-field test or foot shock. Rather, reactive rats showed increased prolactin response to stressors. Prolactin is secreted from the pituitary gland and is most obviously associated with milk production, but experimental studies in humans have found that prolactin also increases in response to social stress (Lennartsson and Jonsdottir 2011). Overall, Blizard et al.'s results indicate that biological stress response involves a complex suite of hormonal systems, not just the HPA axis.

\section{Emerging themes and conclusions}

Considered together, the eight papers in this Special Issue suggest three general considerations for future research. First, as highlighted by Marceau et al. and Van Hulle et al., adrenal and gonadal hormones, such as cortisol, DHEA, and testosterone, have been extensively investigated in behavioral research as putative biological substrates for the effects of environmental stress, challenge, and adversity. Much of this research, however, has not been able to disentangle the hypothesized environmental effects from gene-environment interactions or gene-environment correlations. Moreover, as papers by Van Hulle et al. and Estourgie-van Burk et al. reveal, even relatively basic information on the extent to which naturally-occurring variation in hormonal levels in community samples reflects genetic versus environmental differences has been lacking. As investigators continue to be interested in how environmental experiences, particularly in early life, shape endocrine functioning across the lifespan, there is a pressing need for genetically-informative designs that can deal with potential genetic confounds in order to isolate true environmental effects for behavioral phenotypes. Our field is perfectly poised to address this need.

Second, as illustrated by Ford et al., Tanchuck-Nipper et al., and Blizard et al., the commonalities in hormonal systems across mammalian species provides an excellent opportunity for translational research that bridges experimental designs in animal models and correlational designs in human samples. Like genetically-informative designs, animal research allows for enhanced control in experimental methods and more precise disentangling of environmental, prenatal, and genetic effects. Recent advances in developing animal models with good face validity for behavioral phenotypes in humans have opened the door for novel studies of gene-hormone-behavior interplay. One area that may benefit the most from these advances is the study of how hormones modulate gene expression for behavioral and psychiatric phenotypes. Hormonal manipulations (e.g., gonadectomies) and exogenous hormone exposure (e.g., estrogen administration) are rather straightforward in animals but much more complicated in humans. Likewise, measuring gene expression in the brain is much more straightforward in animals than in humans. Indeed, in animals, changes in gene expression can be directly examined in brain tissue after hormonal manipulations-something that, at present, is impossible to do in humans. It is our hope that investigators will begin to make use of advances in animal behavioral models and experimental technologies (e.g., optogenetics) to explore more fully the complexity of genehormone-behavior interplay.

Finally, we feel compelled to note one major limitation of the papers in this Special Issue. There was a decided lack of racial and ethnic diversity in the participants included, as all samples were overwhelmingly White $(>90 \%)$. There are known racial and ethnic differences in pubertal development (Kaplowitz et al. 2001), in diurnal patterns of cortisol (DeSantis et al. 2015), and in risk for hormonally-related diseases such as ovarian cancer (Goodman et al. 2003) and coronary heart disease 
(Matthews et al. 2006). Moreover, many hormonal systems have been hypothesized to be sensitive to environmental stresses such as perceived racial discrimination (Zeiders et al. 2014). Notably, the papers in this Special Issue are the norm rather than the exception in their homogeneity, as most studies of hormones and genetic factors are predominantly White. There is a critical need for future research on gene-hormone-behavior linkages to include adequate numbers of racial and ethnic minority participants, in order to ensure that results are broadly generalizable and that individual-specific risk factors are elucidated fully.

In conclusion, human behavior is influenced by an array of intersecting hormonal systems that have well-specified genetic underpinnings and that, in many cases, have clear analogues in other mammalian species. This affords behavior genetic research with new opportunities for understanding the links between genotype and behavioral phenotype by considering and incorporating hormones and hormonal change across the lifespan.

\section{References}

Brouwer RM, Koenis MMG, Schnack HG, van Baal GC, van Soelen IL, Boomsma DI, Pol HEH (2015) Longitudinal development of hormone levels and grey matter density in 9 and 12-year-old twins. Behavior Genet. doi:10.1007/s10519-015-9708-8

Cooper GM (2000) The cell: a molecular approach, 2nd edn. Sinauer Associates, Sunderland, MA. Available from: http://www.ncbi. nlm.nih.gov/books/NBK9839/

Corley R, Beltz AM, Wadsworth SJ, Berenbaum SA (2015) Genetic influences on pubertal development and links to behavior problems. Behavior Genetics

DeSantis AS, Adam EK, Hawkley LC, Kudielka BM, Cacioppo JT (2015) Racial and ethnic differences in diurnal cortisol rhythms: are they consistent over time? Psychosom Med 77(1):6-15
Estourgie-van Burk GF, Bartels M, Boomsma DI (2015) A twinsibling study on early growth and hormone levels in adolescents. Behavior Genet. doi:10.1007/s10519-014-9697

Ford MM, Nickel JD, Kaufman MN, Finn DA (2015) Null mutation of $5 \alpha$-reductase type I gene alters ethanol consumption patterns in a sex-dependent manner. Behavior Genet. doi:10.1007/ s10519-014-9694-2

Goodman MT, Howe HL, Tung KH, Hotes J, Miller BA, Coughlin SS, Chen VW (2003) Incidence of ovarian cancer by race and ethnicity in the United States, 1992-1997. Cancer 97(S10): 2676-2685. doi: $10.1002 /$ cncr.11349

Kaplowitz PB, Slora EJ, Wasserman RC, Pedlow SE, HermanGiddens ME (2001) Earlier onset of puberty in girls: relation to increased body mass index and race. Pediatrics 108(2):347-353

Lennartsson AK, Jonsdottir IH (2011) Prolactin in response to acute psychosocial stress in healthy men and women. Psychoneuroendocrinology 36(10):1530-1539

Marceau K, Laurent HK, Neiderhiser JM, Reiss D, Shaw DS, Natsuaki MN, Leve LD (2015) Combined influences of genes, prenatal environment, cortisol, and parenting on the development of children's internalizing versus externalizing problems. Behavior Genet. doi:10.1007/s10519-014-9689-z

Matthews K, Schwartz J, Cohen S, Seeman T (2006) Diurnal cortisol decline is related to coronary calcification: CARDIA study. Psychosom Med 68(5):657-661

Rice F, Harold GT, Boivin J, Hay DF, van den Bree M, Thapar A (2009) Disentangling prenatal and inherited influences in humans with an experimental design. Proc Natl Acad Sci 106(7):2464-2467

Tanchuck-Nipper MA, Ford MM, Hertzberg A, Beadles-Bohling A, Cozzoli DK, Finn DA (2015) Sex differences in ethanol's anxiolytic effect and chronic ethanol withdrawal severity in mice with a null mutation of the $5 \alpha$-reductase type 1 gene. Behavior Genet. doi:10.1007/s10519-014-9691-5

Van Hulle CA, Moore MN, Shirtcliff EA, Lemery-Chalfant K, Goldsmith HH (2015) Genetic and environmental contributions to covariation between dhea and testosterone in adolescent twins. Behavior Genet. doi:10.1007/s10519-015-9709-7

Zeiders KH, Hoyt LT, Adam EK (2014) Associations between selfreported discrimination and diurnal cortisol rhythms among young adults: the moderating role of racial-ethnic minority status. Psychoneuroendocrinology 50:280-288 